\title{
Magnetic phases of bosons with synthetic spin-orbit coupling in optical lattices
}

\author{
Zi Cai, ${ }^{1}$ Xiangfa Zhou, ${ }^{2}$ and Congjun $\mathrm{Wu}^{1,3}$ \\ ${ }^{1}$ Department of Physics, University of California, San Diego, California 92093 \\ ${ }^{2}$ Key Laboratory of Quantum Information, University of Science and Technology of China, CAS, Hefei, Anhui 230026, China \\ 3 Center for Quantum Information, IIIS, Tsinghua University, Beijing, China
}

\begin{abstract}
We investigate magnetic properties in the superfluid and Mott-insulating states of two-component bosons with spin-orbit (SO) coupling in 2D square optical lattices. The spin-independent hopping integral $t$ and SO coupled one $\lambda$ are fitted from band structure calculations in the continuum, which exhibit oscillations as increasing SO coupling strength. The magnetic superexchange model is derived in the Mott-insulating state with one-particle per-site, characterized by the DzyaloshinskyMoriya (DM) interaction. In the limit of $|\lambda| \ll|t|$, we find a spin spiral Mott state whose pitch value is the same as that in the incommensurate superfluid state, while in the opposite limit $|t| \ll|\lambda|$, the ground state can be found by a dual transformation to the $|\lambda| \ll|t|$ limit.
\end{abstract}

PACS numbers: 67.85.Jk, 67.85.Hj, 05.30.Jp

Quantum many-body states with spontaneous incommensurate modulated structure have attracted considerable interests in the past decades, and occur in many settings of condensed matter and ultracold atom physics, such as frustrated magnetism, unconventional superconductor and superfluid and so on. Celebrated examples include the incommensurate magnetism with long-range and short range magnetic order ${ }^{1.2}$, the Fulde-FerrellLarkin-Ovchinnikov (FFLO) pairing states 3,4 . Recently, the Bose-Einstein condensations (BEC) with spin-orbit (SO) coupling introduce a new member to this family. The SO coupled BECs are genuinely new phenomena due to the fact that the kinetic energy is not just a Laplacian but also linearly depends on momentum, which gives rise the complex-valued condensate wavefunctions beyond Feynman's no-node theorem ${ }^{5}$.

An interesting property of SO coupled condensates of bosons is that they can spontaneously break time-reversal symmetry which is absent in conventional BECs of both superfluid ${ }^{4} \mathrm{He}$ and many experiments of ultra-cold alkali bosons 6 . For example, it is predicted that such condensates can spontaneously develop half-quantum vortex coexisting with 2D skyrmion-type spin textures in the harmonic trap ${ }^{7}$. Experimentally, spin textures of the SO coupled bosons have been observed in exciton condensations, which is a solid state boson systems with relativistic SO coupling ${ }^{8}$. Theoretically, extensive studies have been performed for SO coupled bosons which exhibit various spin orderings and textures from competitions among SO coupling, interaction, and confining trap energy $7,11-18$.

In the optical lattice, the SO coupled bosons are even more interesting. Early investigations have showed that the characteristic incommensurate wavevectors are incommensurate with the lattice 19 . In this article, we study the SO coupled Bose-Hubbard model, focusing on the magnetic properties. The tight binding model is constructed and the spin-independent hopping integral $t$ and SO coupled hopping integral $\lambda$ are calculated as functions of the SO coupling strength in the continuum. Magnetic superexchange models are derived character- ized by the Dzyaloshinsky-Moriya (DM) interaction 23,24. In the Mott-insulating phase, single particle condensation is suppressed but the spin order is not. The spin orderings are solved in two different limits, $|\lambda| \ll|t|$, and $|t| \ll|\lambda|$, respectively. In the former case, the DM term destabilizes the ferromagnetic state to spin spirals, while in latter case can be transformed to the former one by a dual transformation .

We begin with the non-interacting Hamiltonian of bosons with the Rashba SO coupling in a square lattice optical potential as

$$
H_{0}=\frac{\hbar^{2} \mathbf{k}^{2}}{2 m} \hat{1}+\frac{\hbar^{2} k_{s o}}{m}\left(\alpha k_{x} \hat{\sigma}_{y}+\beta k_{y} \hat{\sigma}_{x}\right)+V(x, y),
$$

where $k_{s o}$ is the magnitude of wavevectors of laser beams generating SO coupling. $\alpha$ and $\beta$ characterize the anisotropy of SO coupling. Below we consider two situations. First, SO coupling is only along the $x$ direction, i.e., $\alpha=1, \beta=0$, which agrees with the recent experiments ${ }^{10}$. Second, the isotropic Rashba SO coupling with $\alpha=1, \beta=1$. $V(x, y)$ is the periodic potential produced by laser beams with wavelength $\lambda_{0}$ as

$$
V(x, y)=-V_{0}\left[\cos ^{2}\left(k_{0} x\right)+\cos ^{2}\left(k_{0} y\right)\right]
$$

where $k_{0}=2 \pi / \lambda_{0}$, and the recoil energy $E_{r}=\frac{\hbar^{2} k_{0}^{2}}{2 m}$. We define a dimensionless parameter $\gamma_{0}=k_{s o} / k_{0}$ to characterize the strength of SO coupling. The lattice constant $a=\lambda_{0} / 2$, and the reciprocal lattice is $\mathbf{G}_{1}=\left(\frac{2 \pi}{a}, 0\right)$, $\mathbf{G}_{2}=\left(0, \frac{2 \pi}{a}\right)$. The band structure of Eq. 1 is calculated by using the plane-wave basis.

In the absence of SO coupling, the two-component bosons with strong optical potentials can be described by the lattice Bose-Hubbard model as

$$
H_{H u b}=-\sum_{\langle i j\rangle, \sigma} t_{i j}\left[b_{i, \sigma}^{\dagger} b_{j, \sigma}+h . c\right]+\sum_{i}\left[\frac{U}{2} n_{i}^{2}-\mu n_{i}\right]
$$

where $\sigma=\uparrow, \downarrow$ denote the pseudospin components; $b_{i \sigma}$ and $b_{i \sigma}^{\dagger}$ are bosonic annihilation and creation operators 
for spin $\sigma$ at site $i$, respectively. $\sum_{\langle i, j\rangle}$ denotes the summation over all the nearest neighbors. $n_{i}$ is the boson density operator at site $i$ : $n_{i}=\sum_{\sigma} b_{i \sigma}^{\dagger} b_{i \sigma}$. Generally, the interaction can be spin-dependent. In this article, we only consider the spin-independent interaction.

We first consider the case of $\alpha=1, \beta=0$, which is the situation directly related with current experiments in the absence of optical lattice ${ }^{10}$. The SO coupling induces an extra term in the tight-binding term as

$$
H_{s o}=-\lambda \sum_{\mathbf{i}}\left[b_{\mathbf{i}, \uparrow}^{\dagger} b_{\mathbf{i}+\vec{e}_{x}, \downarrow}-b_{\mathbf{i}, \downarrow}^{\dagger} b_{\mathbf{i}+\vec{e}_{x}, \uparrow}\right]+h . c,
$$

where $\vec{e}_{x}$ is the unit vector along the $x$-direction. In momentum space, Eq. 4 becomes $H_{\text {so }}=\sum_{\mathbf{k}} \Psi_{\mathbf{k}}^{\dagger} \hat{H}_{\mathbf{k}} \Psi_{\mathbf{k}}$, where $\Psi_{\mathbf{k}}=\left[b_{\mathbf{k}, \uparrow}, b_{\mathbf{k}, \downarrow}\right]^{T}$, and $\hat{H}_{\mathbf{k}}^{1}$ is a 2 by 2 matrix reads as:

$$
\hat{H}_{\mathbf{k}}=\varepsilon_{\mathbf{k}} \hat{I}+2 \lambda \sin k_{x} \hat{\sigma}_{y}
$$

where $\varepsilon_{\mathbf{k}}=-2\left(t_{x} \cos k_{x}+t_{y} \cos k_{y}\right)-\mu$ and $t_{x}\left(t_{y}\right)$ is the hopping integrals along $x$ and $y$-directions, respectively. In the long-wave limit $k \rightarrow 0$, Eq. (5) reduces to the Hamiltonian in continuous space realized in experiments.

The SO coupling is equivalent to a pure gauge at $\beta=0$, which can be eliminated by a gauge transformation

$$
U=\exp \left\{i \mathbf{k}_{\mathbf{s o}} \cdot \mathbf{r} \sigma_{z}\right\}
$$

which applies to the doublet $\left(b_{\uparrow}, b_{\downarrow}\right)$. The energy spectra of Eq. 5 has two branches as $E_{ \pm}=-2 t_{y}\left[\cos \left(k_{x} \pm k_{s o}\right)+\right.$ $\left.\cos k_{y}\right]-\mu$ corresponding to the eigenvalues \pm 1 of $\sigma_{y}$, and the following relations are satisfied

$$
t_{x}=t_{y} \cos k_{s o}, \quad \lambda=t_{y} \sin k_{s o} .
$$

Bosons condense into the energy minima of $\pm \mathbf{Q}=$ $\left( \pm k_{0}, 0\right)$ with $k_{1}=\arctan \left(\lambda / t_{y}\right)$. The corresponding single particle wavefunctions at these two minima are:

$$
\Psi_{ \pm \mathbf{Q}}=\frac{1}{\sqrt{2}} e^{ \pm i \mathbf{r} \cdot \mathbf{Q}_{s c}}\left(\begin{array}{c}
1 \\
\pm i
\end{array}\right)
$$

At the Hartree-Fock level, bosons can take either of $\Psi_{\mathbf{Q}_{s c}}$ as a plane-wave spin-polarized state, or, a superposition of them as $\frac{1}{\sqrt{2}}\left(\Psi_{\mathbf{Q}}+\Psi_{\mathbf{Q}}\right)=[\cos \mathbf{Q} \cdot \mathbf{r}, \sin \mathbf{Q} \cdot \mathbf{r}]^{T}$ with the same energy. The latter one can be stabilized by spin-dependent interaction of $H_{s p, i n t}=U^{\prime} \sum\left(n_{i, \uparrow}-n_{i, \downarrow}\right)^{2}$ with $U^{\prime}<0$. It exhibits a spin spiral states in the $x z$ plane with the pitch wavevector $2 \mathbf{Q}$ as plotted in Fig. 1] We will see that in the Mott-insulating state, although strong interaction suppresses the superfluidity, the spin configuration remains the same spiral order.

We consider the Mott insulating state at $\left\langle n_{i}\right\rangle=1$, and construct the superexchange Hamiltonian for the pseudospin- $\frac{1}{2}$ bosons as

$$
H_{e f f}=\sum_{\mathbf{i}}\left[H_{\mathbf{i}, \mathbf{i}+\hat{e}_{y}}+H_{\mathbf{i}, \mathbf{i}+\hat{e}_{x}}\right] .
$$

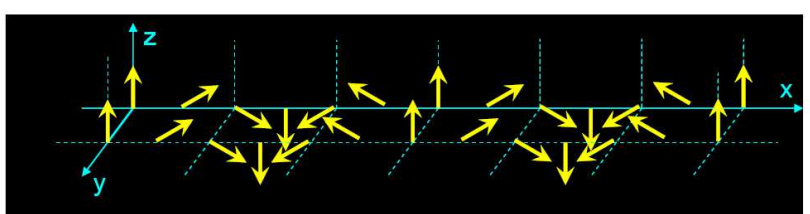

FIG. 1: Spin spiral configurations of the Bose-Hubbard model with unidirectional SO coupling. It is valid for both the incommensurate superfluid state, and the Mott insulating state.

For the vertical bond without SO coupling, $H_{i, i+e_{y}}$ is just the $S U(2)$ ferromagnetic Heisenberg superexchange 25,26 as $H_{\mathbf{i}, \mathbf{i}+\hat{e}_{y}}=-J_{1, y} \mathbf{S}_{\mathbf{i}} \cdot \mathbf{S}_{\mathbf{i}+\hat{e}_{y}}$ where $J_{1, y}=4 t_{y}^{2} / U>$ 0 . For the horizontal bond, the SO coupling leads to the Dzyaloshinsky-Moriya (DM) type superexchange terms 23,24 as

$$
\begin{aligned}
H_{\mathbf{i}, \mathbf{i}+\hat{e}_{x}} & =-J_{1, x} \mathbf{S}_{\mathbf{i}} \cdot \mathbf{S}_{\mathbf{i}+\hat{e}_{x}}-J_{12} \mathbf{d}_{\mathbf{i}, \mathbf{i}+\hat{e}_{x}} \cdot\left(\mathbf{S}_{\mathbf{i}} \times \mathbf{S}_{\mathbf{i}+\hat{e}_{x}}\right) \\
& +J_{2}\left[\mathbf{S}_{\mathbf{i}} \cdot \mathbf{S}_{\mathbf{i}+\hat{e}_{x}}-2\left(\mathbf{S}_{\mathbf{i}} \cdot \mathbf{d}_{\mathbf{i}, \mathbf{i}+\hat{e}_{x}}\right)\left(\mathbf{S}_{\mathbf{i}+\hat{e}_{x}} \cdot \mathbf{d}_{\mathbf{i}, \mathbf{i}+\hat{e}_{x}}\right)\right],
\end{aligned}
$$

where $J_{2}=4 \lambda^{2} / U, J_{12}=4 t_{y} \lambda / U . \mathbf{d}_{\mathbf{i}, \mathbf{i}+\hat{e}_{x}}$ is a $3 \mathrm{D} \mathrm{DM}$ vector defined on the bond $\left[\mathbf{i}, \mathbf{i}+\hat{e}_{x}\right]$, and, $\mathbf{d}_{\mathbf{i}, \mathbf{i}+\hat{e}_{x}}=\hat{e}_{y}$.

The DM term of Eq. (10) prefers a spin spiral ordering along the horizontal direction, as illustrated in Fig[1(b). The effect of the gauge transformation Eq. 6] on spin operators is to rotate $\mathbf{S}_{\mathbf{i}}$ around $y$-axis at the angle of $2 m \theta$ where $m$ is the horizontal coordinate of site $i$ and $\theta=\arctan \left(\lambda / t_{y}\right)^{27}$, such that

$$
\begin{aligned}
\mathbf{S}_{\mathbf{i}}^{\prime} & =(1-\cos 2 m \theta)\left[\mathbf{d} \cdot \mathbf{S}_{\mathbf{i}}\right] \mathbf{d}+\cos 2 m \theta \mathbf{S}_{\mathbf{i}} \\
& -\sin 2 m \theta \mathbf{S}_{\mathbf{i}} \times \mathbf{d}
\end{aligned}
$$

where $\mathbf{d}=\mathbf{d}_{\mathbf{i}, \mathbf{i}+\hat{e}_{x}}=\hat{e}_{y}$. Through this transformation, the DM interaction is gauged away, and Eq.(10) turns into a ferromagnetic coupling:

$$
H_{\mathbf{i}, \mathbf{i}+\hat{e}_{x}}=-J_{0} \mathbf{S}_{\mathbf{i}}^{\prime} \cdot \mathbf{S}_{\mathbf{i}+\hat{e}_{x}}^{\prime}
$$

where $J_{0}=J_{1, y}=4\left(t_{x}^{2}+\lambda^{2}\right) / U$. The the exchange model becomes an isotropic ferromagnetic Heisenberg model, and thus spin polarization can point along any direction. In order to obtain the actual spin spiral configuration, we need to do the inverse operation of Eq. 11. Say, if we choose the classic spin at the point of origin along $z$ direction $\mathbf{S}_{[0,0]}=\hat{e}_{z}$, according to the rotation defined in Eq.111), all the spins in the classic ground state are restricted within the $x-z$ plane, and the classic spin at the point $[m, n]$ is $\mathbf{S}_{[m, n]}=\cos (2 m \theta) \hat{e}_{z}+\sin (2 m \theta) \hat{e}_{x}$. As shown in Fig 1 (b), the classic spins form a chiral pattern with a characteristic length, which is the same as in the superfluid case as plotted in Fig. 11. The only difference is that the superfluid phase coherence is lost in the Mott-insulating state.

Now we discuss the isotropic Rashba SO coupling with $\alpha=\beta=1$. From the symmetry analysis, we easily have 

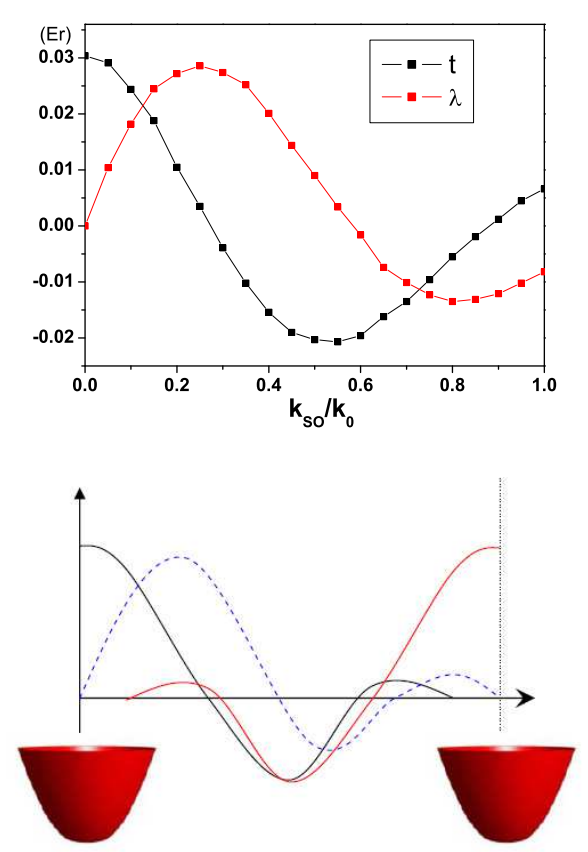

FIG. 2: A) The dependence of the spin-independent hopping integral $t$ and the spin-dependent one $\lambda$ v.s. the SO coupling strength $\gamma=k_{s o} / k_{0}$. The optical potential depth is $V_{0}=8 E_{r}$. B) Sketch of Wannier wavefunctions for $f(r)$ (solid black line) and $g(r)$ (dashed blue line) in Eq. 16.

$t_{x}=t_{y}$ for spin independent hoppings, while the spindependent SO hoppings become

$$
\begin{aligned}
H_{S O}^{\prime} & =-\lambda \sum_{\mathbf{i}}\left[b_{\mathbf{i}, \uparrow}^{\dagger} b_{\mathbf{i}+\vec{e}_{x}, \downarrow}-b_{\mathbf{i}, \downarrow}^{\dagger} b_{\mathbf{i}+\vec{e}_{x}, \uparrow}\right]+h . c, \\
& -i \lambda \sum_{\mathbf{i}}\left[b_{\mathbf{i}, \downarrow}^{\dagger} b_{\mathbf{i}+\vec{e}_{y}, \uparrow}+b_{\mathbf{i}, \uparrow}^{\dagger} b_{\mathbf{i}+\vec{e}_{y}, \downarrow}\right]+h . c .
\end{aligned}
$$

In momentum space, the tight-binding band Hamiltonian turns to: $H^{\prime}=\sum_{\mathbf{k}} \Psi_{\mathbf{k}}^{\dagger} \hat{H}_{\mathbf{k}}^{\prime} \Psi_{\mathbf{k}}$, where

$$
\hat{H}_{\mathbf{k}}^{\prime}=\varepsilon_{\mathbf{k}} \hat{I}+2 \lambda\left[\sin k_{x} \hat{\sigma}_{y}+\sin k_{y} \hat{\sigma}_{x}\right],
$$

where $\varepsilon_{\mathbf{k}}=-2 t\left(\cos k_{x} x+\cos k_{y} y\right)$. The energy spectra of Eq.(14) read

$$
E_{ \pm}^{\prime}=\varepsilon_{\mathbf{k}} \pm 2 \lambda \sqrt{\sin ^{2} k_{x}+\sin ^{2} k_{y}}
$$

The band minima are four-fold degenerate at the points $\mathbf{Q}_{s c}=( \pm k, \pm k)$, where $k=\arctan \frac{\lambda}{\sqrt{2} t}$.

Next we calculate the band parameters $t$ and $\lambda$ versus SO coupling parameter $\gamma$, by fitting the band spectra using the plane-wave basis in the continuum. The results are plotted in Fig. 22 A. Both $t$ and $\lambda$ oscillate and decay as increasing $\gamma$, which can be understood from the behavior of the onsite Wannier functions. Each optical site can be viewed as a local harmonic potential and the lowest single particle state wavefunction was calculated in Ref. [7]

$$
\psi_{j_{z}=\frac{1}{2}}(\vec{r})=\left[f(r), g(r) e^{i \phi}\right]^{T},
$$

and its time-reversal partner is $\psi_{j_{z}=-\frac{1}{2}}(\vec{r})=$ $\left(-g(r) e^{i \phi}, f(r)\right) . f(r)$ and $g(r)$ are real radial wavefunctions, which exhibit characteristic oscillations with the pitch value $k_{s o}$ and a relative phase shift approximately $\frac{\pi}{2}$ as plotted in Fig. 2 B. $t$ and $\lambda$ are related to the off-centered integrals of $f(r)$ and $g(r)$ of two sites, which overlap in the middle. As a result, $t$ and $\lambda$ also oscillate as increasing $\gamma$, which also exhibit a phase shift approximately at $\pi / 2$ as shown in Fig. 2] A.

We would like to clarify one important and subtle point. Actually the on-site Wannier functions are nolonger spin eigenstates, but total angular momentum eigenstate $j_{z}=\frac{1}{2}$, and thus are still a pair of Kramer doublets. For the operators $\left(b_{i \uparrow}, b_{i, \downarrow}\right)^{T}$ defined on site $i$, they do not refer to spin eigenbasis but to the $j_{z^{-}}$ eigenbasis. In fact, in the case that $k_{s o} \geq k_{0}$, the onsite spin moments are nearly zero. The $j_{z}$-movements mainly come from orbital angular momentum. As pointed out in Ref. 7], the Wannier functions of $j_{z}$ eigenstates exhibit skyrmion-type spin texture distributions and halfquantum vortex on each site. This phenomena also remind us of the Friedel oscillation in solid state physics. In the case of $k_{s o} \gg k_{0}$, each site exhibits Landau leveltype quantization: states with different values of $j_{z}$ are nearly degenerate ${ }^{7.18}$, and a single band picture ceases to work here. (a)

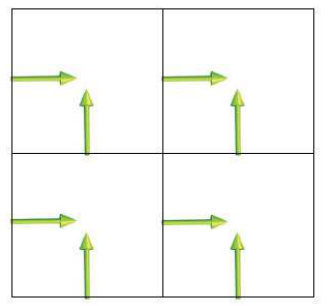

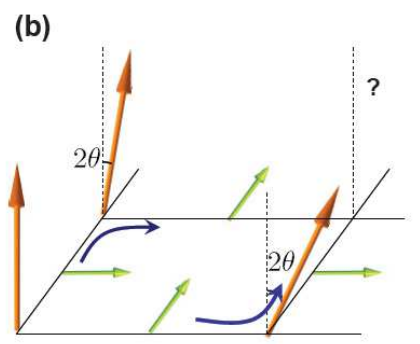

FIG. 3: (A) The pattern of DM vectors of the superexchange magnetic model in the Mott-insulating state. (B) Illustration of the frustration in the spin configuration with DM interactions, the rotations around $x$-axis and $y$-axis do not commutate with each other.

Deep inside the Mott-insulating phase, we obtain the effective magnetic Hamiltonian:

$$
H_{e f f}^{\prime}=\sum_{\mathbf{i}}\left[H_{\mathbf{i}, \mathbf{i}+\hat{e}_{y}}^{\prime}+H_{\mathbf{i}, \mathbf{i}+\hat{e}_{x}}^{\prime}\right] .
$$

$H_{\mathbf{i}, \mathbf{i}+\hat{e}_{x}}^{\prime}$ is the same as Eq.(10), and

$$
\begin{aligned}
& H_{\mathbf{i}, \mathbf{i}+\hat{e}_{y}}^{\prime}=-J_{1} \mathbf{S}_{\mathbf{i}} \cdot \mathbf{S}_{\mathbf{i}+\hat{e}_{y}}-J_{12} \mathbf{d}_{\mathbf{i}, \mathbf{i}+\hat{e}_{y}} \cdot\left(\mathbf{S}_{\mathbf{i}} \times \mathbf{S}_{\mathbf{i}+\hat{e}_{y}}\right) \\
& \quad+J_{2}\left[\mathbf{S}_{\mathbf{i}} \cdot \mathbf{S}_{\mathbf{i}+\hat{e}_{y}}-2\left(\mathbf{S}_{\mathbf{i}} \cdot \mathbf{d}_{\mathbf{i}, \mathbf{i}+\hat{e}_{y}}\right)\left(\mathbf{S}_{\mathbf{i}+\hat{e}_{y}} \cdot \mathbf{d}_{\mathbf{i}, \mathbf{i}+\hat{e}_{y}}\right)\right],
\end{aligned}
$$

where $\mathbf{d}_{\mathbf{i}, \mathbf{i}+\hat{e}_{y}}=\hat{e}_{x}$. The pattern of the DM vectors is shown in Fig 3 (a), which is a strongly reminiscent of that in cuprate superconductor $\mathrm{YBa}_{2} \mathrm{Cu}_{3} \mathrm{O}_{6}{ }^{28,29}$. The classical ground state of Eq. (17) is nontrivial because the $\mathrm{DM}$ interaction can not be gauged away: DM vectors in 
horizontal bonds favor spiraling around the $y$-axis, while that in vertical bonds favor spiraling around the $x$-axis. Since rotations around $x$ and $y$-axis do not commune, no spin configurations can simultaneously satisfy both requirements, which leads to spin frustrations shown in Fig. 3 (B).

The quantum situation of Eq. 17 is even more involved, which can only be solved approximately. Below we focus on two situations: $|\lambda| \ll|t|$ and $|\lambda| \gg|t|$. At $\lambda=0$, the ground state of Eq. (17) is known to be ferromagnetism. At $|\lambda / t| \ll 1$, we use spin-wave approximation to analyze the instability of a ferromagnetic state induced by the DM interaction. Notice that in this case, it is impossible to find a global rotation as in Eq. (11) to gauge away the DM vectors and transform Eq. (17) to an $S O(3)$ invariant Hamiltonian, thus the quantized axis in the spin wave analysis can not be chosen arbitrarily. To gain some insight, we choose a classic ferromagnetic state as a variational ground state parameterized by $\mathbf{S}_{\mathbf{i}}=S(\cos \gamma \sin \eta, \sin \gamma \sin \eta, \cos \eta)$. The corresponding variational energy $E_{0}=-S^{2}\left(J_{1}-J_{2}+2 \sin ^{2} \eta J_{2}\right)$ is minimized when $\eta=\pi / 2$, which implies that the $x y$ plane is the easy plane.

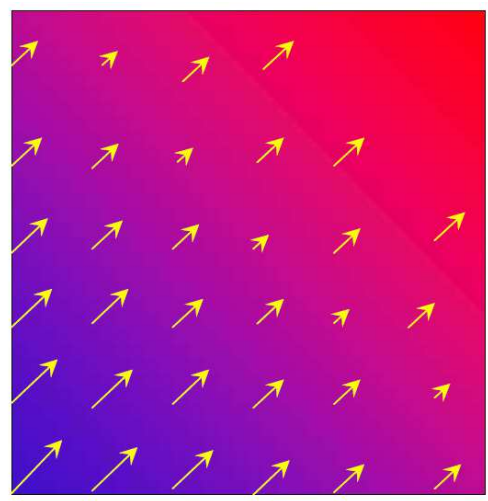

FIG. 4: (Spin spiral ordering in the limit of $|\lambda| \ll|t|$.

To calculate the spin waves spectra, it is convenient to rotate the coordinate so that the new $z$-axis points along the direction $\mathbf{l}=[\overline{1} \overline{1} 0]$ in the original coordinate (we choose $\mathbf{l}$ as the quantized axis). The Holstein-Primakoff transformation is employed to transform Eq. (17) into the bosonic Hamiltonian:

$$
H_{b}=\sum_{i, \mu}-J_{0}(\cos 2 \theta-i \sin 2 \theta / \sqrt{2}) a_{i}^{\dagger} a_{i+e_{\mu}}+h . c,
$$

where $\theta=\arctan (\lambda / t)$ as defined above, $\mu=x, y$. We only keep quadric terms and ignore the terms proportional to $\sin ^{2} \theta$ since $\lambda / t \ll 1$. In momentum space, it becomes

$$
\begin{aligned}
H_{e x}^{\prime} & =-2 J_{0} \sum_{\mathbf{k}}\left[\cos 2 \theta \cos k_{x}+\frac{1}{\sqrt{2}} \sin 2 \theta \sin k_{x}\right. \\
& \left.+\cos 2 \theta \cos k_{y}+\frac{1}{\sqrt{2}} \sin 2 \theta \sin k_{y}\right] c_{\mathbf{k}}^{\dagger} c_{\mathbf{k}},
\end{aligned}
$$

The minimum of the dispersion of Eq. 20 occurs at points $\mathbf{Q}_{M}=\left( \pm k^{\prime}, \pm k^{\prime}\right)$, where $k^{\prime}$ satisfies that $\tan k^{\prime}=$ $\frac{1}{\sqrt{2}} \tan 2 \theta$. Compare it with the energy minima in the noninteracting band Hamiltonian $\mathbf{Q}_{s f}=( \pm k, \pm k)$, we have $k^{\prime}=2 k$ at the limit of $\gamma \rightarrow 0$. The nonzero minimum of the magnon spectrum is a signature of the spin spiral order, as shown in Fig. 4 (a).

Interestingly, in the opposite limit of $|\lambda / t| \gg 1$, Eq. (10) $)$ can be related to that of $|\lambda / t| \ll 1$ through a duality transformation. On site $i$ with the coordinates $\left(i_{x}, i_{y}\right)$, $\vec{S}_{i}$ is transformed into

$$
\begin{aligned}
& S_{i_{x}, i_{y}}^{x} \rightarrow(-1)^{i_{x}} \mathbb{S}_{i_{x}, i_{y}}^{x} ; \quad S_{i_{x}, i_{y}}^{y} \rightarrow(-1)^{i_{y}} \mathbb{S}_{i_{x}, i_{y}}^{y} \\
& S_{i_{x}, i_{y}}^{z} \rightarrow(-1)^{i_{x}+i_{y}} \mathbb{S}_{i_{x}, i_{y}}^{z} .
\end{aligned}
$$

$\overrightarrow{\mathbb{S}}_{i}$ still maintains the spin commutation relation. Under this transformation, the $J_{1}$-term transforms into the $J_{2}$ term and vice versa, and the $J_{12}$-term is invariant. Thus this dual transformation indicates that there is a oneto-one correspondence between the $J_{2}$-dominant phase $(|\lambda / t| \gg 1)$ and that of $J_{1}$ with $|\lambda / t| \ll 1$ which has been analyzed above.

In conclusion, we have investigated the magnetic ordering of two-component Bose-Hubbard model with synthetic SO coupling. The band parameters of hopping integrals exhibit characteristic oscillations as increasing SO coupling strength, and the onsite magnetic moments are nearly orbital moments at large SO coupling strength. In the Mott-insulating state with one particle per site, an effective magnetic superexchange model with the DM type interaction is derive. The spin spiral state and its dual state are found in the limits of $|\lambda| \ll|t|$ and $|\lambda| \gg|t|$.

This work was supported by the NSF DMR-1105945, the AFOSR-YIP program.

Note added Up the posting of this paper, we become aware two papers on the similar topic 30,31 .
1 N. Read and S. Sachdev, Phys. Rev. Lett. 66, 1773 (1991).

2 S. Sachdev and N. Read, Mod. Phys. Lett. 5, 219 (1991).

3 P. Fulde and R. A. Ferrell, Phys. Rev. 135, A550 (1964).

4 A. I. Larkin and Y. N. Ovchinnikov, Sov. Phys.-JETP 20, 762 (1965).

5 R. P. Feynman, Statistical Mechanics, A Set of Lectures (Berlin: Addison-Wesley, 1972).
6 A. J. Leggett, Rev. Mod. Phys. 73, 307 (2001).

7 C. Wu , I. Mondragon-Shem, arXiv:0809.3532 C. Wu , I. Mondragon-Shem, and X. F. Zhou, Chin. Phys. Lett., 28, 097102 (2011).

8 A.A. High et al., Nature 483, 584 (2012). A.A. High et al., arXiv:1103.0321

9 Y.-J. Lin et al., Nature 462, 628 (2009). 
10 Y.-J. Lin, K. Jiménez-García and I. B. Spielman, Nature 471, 83 (2011).

11 T. Stanescu, B. Anderson, V. Galitski Phys. Rev. A 78, 023616 (2008).

12 T.-L. Ho and S. Zhang, Phys. Rev. Lett. 107, 150403 (2011).

13 C. Wang, C. Gao, C.M. Jian, H. Zhai, Phys. Rev. Lett. 105, 160403 (2010).

14 S.-K. Yip, Phys. Rev. A 83, 043616 (2011).

15 Y. Zhang, L. Mao, and C. Zhang, Phys. Rev. Lett. 108, 035302 (2012).

16 X.-F. Zhou, J. Zhou, and C. Wu, Phys. Rev. A 84, 063624 (2011).

17 Y. Li, X. F. Zhou, and C. Wu, arXiv:1205.2162

18 H. Hu, B. Ramachandhran, H. Pu, and X.J Liu, Phys. Rev. Lett. 108, 010402(2012).

19 I. Mondragon-shem, B. A. Rodriguez, C. Wu, Bull. Am. Phys. Soc. 55, MAR.Z31.11 (2010). I. Mondragon-shem, Bachelor thesis, Instituto de FAsica, Facultad de Ciencias Exactas y Naturales, Universidad de Antioquia (2010).
20 S. Sinha, R. Nath, and L. Santos, arXiv:1102.2045.

21 Y. Li, X. Zhou, and C. Wu, Phys. Rev. B 85, 125122 (2012).

22 S.K. Ghosh, J.P. Vyasanakere, V.B. Shenoy, Phys. Rev. A, 84, 053629 (2011).

23 I. Dzyaloshinsky, J. Phys. and Chem. Sol. 4, 241 (1958).

${ }^{24}$ T. Moriya, Phys. Rev. 120, 91 (1960).

25 L. M. Duan, E. Demler, and M. D. Lukin, Phys. Rev. Lett. 91, 090402 (2003).

26 A. B. Kuklov and B. V. Svistunov, Phys. Rev. Lett. 90, 100401 (2003).

27 L. Shekhtman, O. Entin-Wohlman, and A. Aharony, Phys. Rev. Lett. 69, 836 (1992).

28 D. Coffey, T. M. Rice, and F. C. Zhang, Phys. Rev. B 44, 10112 (1991).

29 N. E. Bonesteel, Phys. Rev. B 47, 11302 (1993).

30 J. Radic, A. Di Ciolo, K. Sun, V. Galitski, arXiv:1205.2110.

31 W. S. Cole, S. Z. Zhang, A. Paramekanti, and N Trivedi, arXiv:1205.2319. 